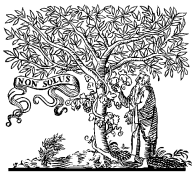

\title{
The BDI-II factor structure in pregnancy and postpartum: Two or three factors?
}

\author{
Sandra Carvalho Bos ${ }^{\text {a,* }}$, Ana Telma Pereira ${ }^{\text {a }}$, Mariana Marques ${ }^{\text {a }}$, Berta Maia a , \\ Maria João Soares ${ }^{a}$, José Valente ${ }^{\text {a }}$, Ana Gomes ${ }^{\text {b,1 }}$, António Macedo ${ }^{a}$, Maria Helena Azevedo ${ }^{a}$ \\ a Institute of Medical Psychology, Faculty of Medicine, University of Coimbra, Rua Larga, 3004-504, Coimbra, Portugal \\ ${ }^{\mathrm{b}}$ Department of Educational Sciences, University of Aveiro, Campus de Santiago, 3810-193, Aveiro, Portugal
}

Received 5 May 2008; received in revised form 1 October 2008; accepted 14 October 2008

\begin{abstract}
The purpose of the present study was to investigate the factor structure of the Beck Depression Inventory-II (BDI-II) in pregnancy and postpartum. Women were asked to fill in the BDI-II in their last trimester of pregnancy and at 3 months after delivery. A total of 331 pregnant women, with a mean age of 29.7 years $(\mathrm{SD}=4.6)$, and 354 mothers, aged 30.6 years $(\mathrm{SD}=4.6$ years), answered the BDI-II. The first group was mainly nulliparas $(65.6 \%)$ and the second group was mostly primiparas $(57.4 \%)$. Factor analyses with principal components solution and varimax rotation were performed. Based on the scree test of Cattell a 2-factor solution and a 3-factor solution were explored. The 2-factor solution was identical in pregnancy and postpartum. Items loading in the Cognitive-Affective factor and in the Somatic-Anxiety factor were almost the same, though the Cognitive-Affective factor explained more of the BDI-II total variance in pregnancy, whereas in postpartum both factors explained similar total variances. The 3-factor solution of the BDI-II in pregnancy and postpartum slightly diverged. Besides the Cognitive-Affective and the Somatic-Anxiety factors, a third factor, Fatigue, was obtained in pregnancy while Guilt was the third factor identified in postpartum. This study reveals that the BDI-II 3-factor solution might be more appropriate to assess depressive symptoms in pregnancy and postpartum.
\end{abstract}

(C) 2008 Elsevier Masson SAS. All rights reserved.

Keywords: Psychometric and assessments in psychiatry; BDI-II factor analyses; Pregnancy; Postpartum

\section{Introduction}

The Beck Depression Inventory [5] is one of the most widely used self-report instruments to evaluate depressive symptoms both in clinical and non-clinical populations.

The last version of the BDI (BDI-II) [4] was designed to accommodate the changes that occurred in Major Depression Criteria as defined in DSM-IV [1]. The total score is the sum of the 21 items' ratings and the maximum total score possible is 63. A cut-off score of 13 is used to help detecting Depression. Higher scores indicate more severe Depression [4].

\footnotetext{
* Corresponding author. Tel.: +351 239 857700; fax: +351 239823170

E-mail address: sbos@fmed.uc.pt (S. Carvalho Bos).

${ }^{1}$ Tel.: +351 234 370628; fax: +351 234370640 .
}

The BDI-II structure has been explored in several studies using factor analysis [30]. This method can identify factors that may represent dimensions or concepts of a more broad conceptual system. For example, in Depression, underlying constructs can emerge as factors, which in turn may help to contribute for an explanatory theory of mood disorders [14].

A factor structure of the BDI-II with a 2-factor solution is frequently obtained in clinical samples $[4,27,28]$ and nonclinical samples $[29,31]$. The 2 -factor solution in psychiatric patients $[4,27,28]$ usually comprises, first, a Somatic-affective dimension (Factor 1) and, secondly, a Cognitive factor (Factor 2). This structure confirms the relevance of Somaticaffective symptoms in clinical populations. In non-clinical samples (mostly students) the 2-factor solution model slightly differs from the one mentioned above [30]. The CognitiveAffective factor is usually the first factor identified and the 
Somatic factor is the second factor obtained [4,29,31]. The term "Affective" which is normally associated with the Somatic factor in the 2-factor model of clinical samples, usually is linked to the Cognitive factor, in non-clinical samples. In fact, Ward [30] refers that affective symptoms such as sadness or crying are likely to shift from one factor to the other depending on the sample being investigated.

To our knowledge, the factor structure of the BDI-II has not yet been explored in pregnancy. There is a study carried out by Salamero et al. [26] with a large sample of non-psychiatric pregnant women $(N=882)$ but the authors used the original BDI. The factor structure obtained was similar to the one often achieved with non-clinical samples: Factor 1, CognitiveAffective, included items such as sense of punishment or selfdislike; Factor 2, Somatic and Inhibition factor, included items such as work inhibition or fatigability.

In postpartum, a 3-factor solution has also been reported using a sample of recently new mothers [19]. Mahmud et al. [19] explored the factor analysis of the BDI-II within a nonclinical sample of 354 women (4-12 weeks after delivery). The sample was selected from health centres from Kota Setar and Pendang districts in Kedah using a systematic random sampling procedure. The authors identified 3 factors: an Affective factor that included items as agitation, crying and irritability, a Somatic factor with items such as tiredness and loss of energy and a Cognitive factor composed by items such as punishment feelings and worthlessness.

Although Motherhood is a normal phase of a woman's life, there is a risk of developing a number of physical and psychological problems, particularly emotional distress of various forms and severity [24]. Moreover, recent scientific literature and studies refer the importance of considering the entire perinatal period from pregnancy to postpartum, and not only postpartum, as depression and anxiety often begin in pregnancy $[9,13,17]$.

Factor analyses of the BDI-II could contribute to investigate the nature of disordered mood experienced by women at these times, unravelling symptoms of Depression which are often veiled by somatic complaints. Thus, as the BDI-II factor structure has not been completely investigated in pregnancy and postpartum the aim of the present study was to explore this issue in a non-clinical population.

\section{Subjects and methods}

Data for this study were drawn from a research project funded by the Portuguese Science and Technology Foundation, FCT (POCI/SAU-ESP/57068/2004). The project was approved by the Ethical Committee of the Faculty of Medicine, Coimbra.

Healthy pregnant women in their last trimester of gestation, following their pregnancy at Local Health Medical Centres, while waiting for their prenatal medical appointment, were invited to participate in the study. This recruitment procedure of collecting a sample from the general population had the objective of avoiding the selection of subgroups with high risk pregnancies (these are followed in obstetric units) and also circumvent the "help seeking behaviour" bias, since it is recognized that persons who seek treatment may be unrepresentative of those who are unwilling or unable to seek treatment [18].

After delivery, women who already had taken part in the study or who had given their written consent to participate when entering the Local Maternity to give birth were contacted by phone 3 months after delivery to set up an appointment. These meetings usually took place at the mother's Medical Centre (frequently when mothers took their babies for vaccination) or at their homes.

Aims and procedures were explained to participants, confidentiality was guaranteed and written consent was obtained. After their approval women were asked to complete a battery of tests including the Portuguese version of the BDIII in pregnancy and postpartum [10,20]. They were also interviewed with the Portuguese version of the Diagnostic Interview for Genetic Studies (DIGS), which has shown excellent inter-rater reliability [3,22]. The DIGS allows the user to identify symptoms of different psychiatric disorders, namely Depression, and to undertake diagnosis according to different diagnostic systems including DSM-IV [1] and ICD10 [32].

Only Demographics, Medical History, Overview of Psychiatric Disturbance, Major Depression, Dysthymia, Depressive/Hyperthymic Personality, Suicidal Behaviour sections, and a brief section of Mania/Hypomania of DIGS were explored in pregnancy. Sections on Major Depression, Suicidal Behaviour and a reduced part of the Mania/Hypomania section of DIGS were assessed in postpartum.

To obtain final consensus diagnoses of Depression, the best-estimate diagnostic procedure was applied, following the process described elsewhere [2]. The interviewer and an independent rater, based upon the information obtained from the DIGS [2,21], filled the Operational Criteria Checklist for Psychotic Illness (OPCRIT). The data from this checklist were entered in the OPCRIT software system, and the diagnoses were generated. The OPCRIT is a polydiagnostic system that produces psychiatric diagnoses according to 13 operational diagnostic criteria systems, but only the DSM-IV and ICD-10 were used in the present study.

Lifetime prevalence of Major Depression/DSM-IV was $36.4 \%$ and $42.1 \%$ according to ICD-10 (including Mild Depression, Mild Depression with Somatic Syndrome, Moderate Depression with Somatic Syndrome, Severe Depression without Psychotic symptoms and Severe Depression with Psychotic symptoms).

The prevalence of current (considering the previous month before the interview assessment) Major Depression/DSM-IV in pregnancy was $1.5 \%$ and $3.3 \%$ based on ICD-10 classification system. In postpartum the prevalence of current Depression (previous month) was 3.9\%, according to DSM-IV, and $4.6 \%$, based on ICD-10 criteria.

As the purpose of the study was to explore the factor structure of BDI-II in pregnancy and postpartum based on samples that reflected the community population, in an effort to avoid a "totally healthy or pure" and, therefore, biased 
sample, women with lifetime depression were included, even if currently depressed.

Three hundred and thirty-one pregnant women with a mean age of 29.7 years $(\mathrm{SD}=4.6$; Range $=19-42$ years $)$ who were in their last trimester of pregnancy $(M=32.7, \mathrm{SD}=3.4$, Range $=26-41$ weeks of gestation), mostly nulliparous $(65.6 \%)$, participated in the study. At 3 months postpartum 170 women from the sample mentioned above and other new 184 mothers also took part in the study. In total a sample of 354 mothers with a mean age of 30.6 years $(\mathrm{SD}=4.6$, Range $=18-44$ years), $57.4 \%$ primiparous was obtained.

Approximately $8 \%$ of the women refused to participate (the most common reasons given for refusal were not having enough time or being from far-away places). Since data were collected by researchers and not by clinical staff, information about the demographic or clinical attributes of the nonrespondents was not available.

BDI-II factor analyses using the principal components solution with varimax rotation were conducted (Tables 1-4). The Cronbach's alpha was computed as a measure of the internal consistency of the scale and of its factors. High values indicate consistent and reliable measures $(0.65-0.70$, acceptable; $0.70-0.80$, good; $0.80-0.90$, very good). Cronbach's alpha inferior to 0.60 is considered unacceptable reliability

Table 1

Rotated factor matrix of the BDI-II in Pregnancy $(N=331)-2$ factors

\begin{tabular}{|c|c|c|}
\hline \multicolumn{3}{|l|}{ Pregnancy } \\
\hline \multirow[t]{2}{*}{ Factors/Symptoms } & \multicolumn{2}{|l|}{ Loadings } \\
\hline & Factor 1 & Factor 2 \\
\hline \multicolumn{3}{|c|}{ Cognitive-Affective (TVE $=26.5 \%$ ) } \\
\hline Punishment Feelings & 0.744 & 0.013 \\
\hline Past Failure & 0.707 & 0.194 \\
\hline Pessimism & 0.706 & 0.129 \\
\hline Self-Criticalness & 0.701 & 0.232 \\
\hline Sadness & 0.664 & 0.269 \\
\hline Loss of Interest & 0.659 & 0.232 \\
\hline Indecisiveness & 0.657 & 0.301 \\
\hline Worthlessness & 0.641 & 0.123 \\
\hline Loss of Pleasure & 0.623 & 0.259 \\
\hline Self-Dislike & 0.569 & 0.321 \\
\hline Guilty Feelings & 0.543 & 0.151 \\
\hline Suicidal Thoughts or Wishes & 0.493 & -0.055 \\
\hline \multicolumn{3}{|l|}{ Somatic-Anxiety $(\mathrm{TVE}=15.5 \%)$} \\
\hline Tiredness or Fatigue & -0.002 & 0.717 \\
\hline Loss of Energy & 0.192 & 0.661 \\
\hline Irritability & 0.382 & 0.613 \\
\hline Changes in Sleeping Pattern & 0.027 & 0.593 \\
\hline Agitation & 0.337 & 0.539 \\
\hline Changes in Appetite & 0.026 & 0.460 \\
\hline Crying & 0.282 & 0.436 \\
\hline Concentration Difficulty & 0.412 & 0.432 \\
\hline Loss of Interest in Sex & 0.135 & 0.409 \\
\hline \multicolumn{3}{|l|}{ Total Variance Explained $=42.0 \%$} \\
\hline \multicolumn{3}{|l|}{ Correlation between factors } \\
\hline Factor I & - & \\
\hline Factor II & 0.56 & - \\
\hline
\end{tabular}

Item loadings for each factor are shown in italics.

$\mathrm{TVE}=$ total variance explained.
Table 2

Rotated factor matrix of the BDI-II in Postpartum $(N=354)-2$ factors.

\begin{tabular}{|c|c|c|}
\hline \multicolumn{3}{|l|}{ Postpartum } \\
\hline \multirow[t]{2}{*}{ Factors/Symptoms } & \multicolumn{2}{|l|}{ Loadings } \\
\hline & Factor 1 & Factor 2 \\
\hline \multicolumn{3}{|l|}{ Somatic-Anxiety $(\mathrm{TVE}=21.4 \%)$} \\
\hline Tiredness or Fatigue & 0.757 & 0.115 \\
\hline Loss of Energy & 0.723 & 0.106 \\
\hline Concentration Difficulty & 0.685 & 0.234 \\
\hline Irritability & 0.678 & 0.221 \\
\hline Changes in Sleeping Pattern & 0.638 & 0.060 \\
\hline Agitation & 0.590 & 0.387 \\
\hline Indecisiveness & 0.511 & 0.338 \\
\hline Changes in Appetite & 0.509 & 0.140 \\
\hline Crying & 0.485 & 0.393 \\
\hline Loss of Interest in Sex & 0.483 & 0.219 \\
\hline \multicolumn{3}{|c|}{ Cognitive-Affective (TVE $=21.3 \%$ ) } \\
\hline Loss of Pleasure & 0.309 & 0.686 \\
\hline Worthlessness & 0.252 & 0.682 \\
\hline Loss of Interest & 0.333 & 0.661 \\
\hline Guilty Feelings & 0.043 & 0.657 \\
\hline Pessimism & 0.309 & 0.656 \\
\hline Sadness & 0.384 & 0.653 \\
\hline Past Failure & 0.008 & 0.635 \\
\hline Self-Dislike & 0.181 & 0.589 \\
\hline Self-Criticalness & 0.397 & 0.455 \\
\hline Punishment Feelings & 0.085 & 0.354 \\
\hline Suicidal Thoughts or Wishes & 0.085 & 0.342 \\
\hline \multicolumn{3}{|l|}{ Total Variance Explained $=42.7 \%$} \\
\hline \multicolumn{3}{|l|}{ Correlation between factors } \\
\hline Factor I & - & \\
\hline Factor II & 0.51 & - \\
\hline
\end{tabular}

Item loadings for each factor are shown in italics.

$\mathrm{TVE}=$ total variance explained.

according to DeVellis [12]. The Spearman's rank correlation coefficient was used to determine the magnitude of association between factors. The interpretation of the association was based on the criteria defined by Cohen [11] for coefficient correlations: $0.01=$ small; $0.30=$ medium, and $0.50=$ large.

\section{Results}

Based on the scree test of Cattell 2-factor structures were considered: one with a 2-factor solution and the other with a 3factor solution.

\subsection{The 2-factor solution}

In pregnancy the 2 -factor solution explained $42.0 \%$ of the total variance:

- Factor 1 (Cognitive-Affective) - accounted for $26.5 \%$ of the total variance and included 12 symptoms: punishment feelings, past failure, pessimism, self-criticalness, sadness, loss of interest, indecisiveness, worthlessness, loss of pleasure, self-dislike, guilty feelings, and suicidal thoughts.

- Factor 2 (Somatic-Anxiety) - accounted for $15.5 \%$ of the total variance and included 9 items: tiredness or fatigue, 
Table 3

Rotated factor matrix of the BDI-II in Pregnancy $(N=331)-3$ factors.

\begin{tabular}{|c|c|c|c|}
\hline \multicolumn{4}{|l|}{ Pregnancy } \\
\hline \multirow[t]{2}{*}{ Factors/Symptoms } & \multicolumn{3}{|l|}{ Loadings } \\
\hline & Factor I & Factor II & Factor III \\
\hline \multicolumn{4}{|c|}{ Cognitive - Affective $(\mathrm{TVE}=25.8 \%$ ) } \\
\hline Punishment Feelings & 0.737 & 0.166 & -0.106 \\
\hline Past Failure & 0.701 & 0.105 & 0.111 \\
\hline Pessimism & 0.700 & 0.157 & 0.150 \\
\hline Self-Criticalness & 0.696 & 0.121 & 0.237 \\
\hline Indecisiveness & 0.652 & 0.159 & 0.199 \\
\hline Loss of Pleasure & 0.648 & 0.348 & 0.071 \\
\hline Sadness & 0.646 & 0.242 & 0.215 \\
\hline Worthlessness & 0.641 & -0.005 & 0.204 \\
\hline Loss of Interest & 0.623 & -0.001 & 0.388 \\
\hline Guilty Feelings & 0.559 & 0.213 & 0.267 \\
\hline Self-Dislike & 0.536 & 0.179 & 0.062 \\
\hline Suicidal Thoughts or Wishes & 0.493 & 0.019 & -0.071 \\
\hline \multicolumn{4}{|l|}{ Anxiety $(\mathrm{TVE}=11.2 \%)$} \\
\hline Crying & 0.249 & 0.720 & -0.072 \\
\hline Agitation & 0.307 & 0.649 & 0.141 \\
\hline Irritability & 0.353 & 0.617 & 0.276 \\
\hline Changes in Appetite & 0.001 & 0.527 & 0.132 \\
\hline Changes in Sleeping Pattern & 0.006 & 0.440 & 0.402 \\
\hline \multicolumn{4}{|l|}{ Fatigue $(\mathrm{TVE}=10.9 \%)$} \\
\hline Tiredness or Fatigue & -0.019 & 0.335 & 0.672 \\
\hline Loss of Energy & 0.177 & 0.292 & 0.643 \\
\hline Loss of Interest in Sex & 0.135 & -0.037 & 0.607 \\
\hline Concentration Difficulty & 0.409 & 0.055 & 0.564 \\
\hline \multicolumn{4}{|l|}{ Total Variance Explained $=47.9 \%$} \\
\hline \multicolumn{4}{|l|}{ Correlation between factors } \\
\hline Factor I & - & & \\
\hline Factor II & 0.47 & - & \\
\hline Factor III & 0.51 & 0.47 & - \\
\hline
\end{tabular}

Item loadings for each factor are shown in italics.

$\mathrm{TVE}=$ total variance explained.

loss of energy, irritability, changes in sleeping pattern, agitation, changes in appetite, crying, concentration difficulty, and loss of interest in sex (see Table 1).

The correlation between factors was $r_{\mathrm{s}}=0.56$ suggesting a strong association between factors.

In postpartum the 2-factor solution explained $42.7 \%$ of the total variance.

- Factor 1 (Somatic-Anxiety) explained $21.4 \%$ of the total variance and included symptoms such as tiredness or fatigue, irritability, changes in sleeping pattern or appetite, agitation and crying.

- Factor 2 (Cognitive-Affective) explained $21.3 \%$ of the total variance and integrated symptoms as loss of pleasure or interest, pessimism, sadness and self-dislike.

The correlation between factors was also strong $r_{\mathrm{s}}=0.51$, (see Table 2).

With respect to the BDI-II internal consistency the Cronbach's alpha for the total scale was $\alpha=0.875$ in pregnancy
Table 4

Rotated factor matrix of the BDI-II in Postpartum $(N=354)-3$ factors.

\begin{tabular}{|c|c|c|c|}
\hline \multicolumn{4}{|l|}{ Postpartum } \\
\hline \multirow{2}{*}{ Factors/Symptoms } & \multicolumn{3}{|l|}{ Loadings } \\
\hline & Factor I & Factor II & Factor III \\
\hline \multicolumn{4}{|c|}{ Cognitive-Affective (TVE $=20.6 \%)$} \\
\hline Sadness & 0.730 & 0.309 & 0.068 \\
\hline Loss of Interest & 0.724 & 0.260 & 0.089 \\
\hline Loss of Pleasure & 0.698 & 0.251 & 0.186 \\
\hline Worthlessness & 0.673 & 0.199 & 0.217 \\
\hline Pessimism & 0.667 & 0.253 & 0.180 \\
\hline Self-Dislike & 0.593 & 0.131 & 0.163 \\
\hline Self-Criticalness & 0.500 & 0.351 & 0.076 \\
\hline $\begin{array}{l}\text { Suicidal Thoughts } \\
\text { or Wishes }\end{array}$ & 0.454 & 0.020 & -0.106 \\
\hline \multicolumn{4}{|l|}{ Somatic-Anxiety $(\mathrm{TVE}=19.8 \%)$} \\
\hline Tiredness or Fatigue & 0.178 & 0.746 & -0.004 \\
\hline Loss of Energy & 0.200 & 0.702 & -0.066 \\
\hline Concentration Difficulty & 0.263 & 0.671 & 0.077 \\
\hline Changes in Sleeping Pattern & 0.039 & 0.655 & 0.118 \\
\hline Irritability & 0.361 & 0.630 & -0.126 \\
\hline Changes in Appetite & -0.027 & 0.555 & 0.315 \\
\hline Agitation & 0.441 & 0.552 & 0.063 \\
\hline Indecisiveness & 0.294 & 0.507 & 0.218 \\
\hline Loss of Interest in Sex & 0.187 & 0.485 & 0.164 \\
\hline Crying & 0.385 & 0.464 & 0.167 \\
\hline \multicolumn{4}{|l|}{ Guilt (TVE $=9.0 \%)$} \\
\hline Punishment Feelings & -0.53 & 0.185 & 0.832 \\
\hline Past Failure & 0.334 & 0.047 & 0.706 \\
\hline Guilty Feelings & 0.462 & 0.047 & 0.524 \\
\hline \multicolumn{4}{|l|}{ Total Variance Explained $=49.4 \%$} \\
\hline \multicolumn{4}{|l|}{ Correlation between factors } \\
\hline Factor I & - & & \\
\hline Factor II & 0.56 & - & \\
\hline Factor III & 0.30 & 0.20 & - \\
\hline
\end{tabular}

Item loadings for each factor are shown in italics.

$\mathrm{TVE}=$ total variance explained.

and $\alpha=0.889$ postpartum. The Cronbach's alpha in pregnancy for the Cognitive-Affective dimension was $\alpha=0.885$ and for the Somatic-Anxiety dimension was $\alpha=0.747$. In postpartum the Cronbach's alpha for the Somatic-Anxiety dimension was higher in comparison to the value observed in pregnancy, $\alpha=0.842$, and for the Cognitive-Affective dimension was $\alpha=0.842$. All these values indicate good reliabilities [12].

\subsection{The 3-factor solution}

The 3-factor solution in pregnancy was similar to the 2factor solution except that the Somatic-Anxiety factor was divided in an Anxiety factor, F2, which included the crying, agitation, irritability, changes in appetite and changes in sleeping pattern items and in a Fatigue factor, F3, with items as tiredness or fatigue, loss of energy, loss of interest in sex, and concentration difficulty. The 3 factors explained $47.9 \%$ of the total variance (Table 3). F1, the Cognitive-Affective factor, explained $25.8 \%$ of the total variance whereas F2 and F3 accounted for $11.2 \%$ and $10.9 \%$, respectively. Spearman 
correlation between $\mathrm{F} 1$ and F2 was $r_{\mathrm{s}}=0.47$, between F2 and $\mathrm{F} 3$ was $r_{\mathrm{s}}=0.47$ and between F1 and F3 was $r_{\mathrm{s}}=0.51$ (see Table 3). These coefficients suggest a strong correlation between factors.

In postpartum the 3-factor solution of the BDI-II explained $49.4 \%$ of the total variance and included:

- Factor 1, F1 (Cognitive-Affective), included symptoms related to Cognitive and Affective dimensions (explaining $20.6 \%$ of the total variance);

- Factor 2, F2 (Somatic-Anxiety) which remained equal to Factor 1 from the previous 2-factor structure in postpartum (explaining $19.8 \%$ of the total variance);

- Factor 3, F3 (Guilt) included symptoms of guilt, punishment and past failure feelings (explaining 9\% of the total variance).

The correlation between F1 and F2 was $r_{\mathrm{s}}=0.56$, between $\mathrm{F} 2$ and $\mathrm{F} 3$ was $r_{\mathrm{s}}=0.20$ and between $\mathrm{F} 1$ and $\mathrm{F} 3$ was $r_{\mathrm{s}}=0.30$ (see Table 4). These results indicate a strong correlation between F1 and F2, a small correlation between F2 and F3 and a moderate correlation between F1 and F3.

Considering the internal consistency for the 3-factor BDI-II solution, it was observed in pregnancy that the Cronbach's alpha was $\alpha=0.885$ for the Cognitive-Affective factor, $\alpha=0.667$ for the Anxiety factor and $\alpha=0.645$ for the Somatic factor. In postpartum the Cronbach's alpha was $\alpha=0.837$ for the Cognitive-Affective factor, $\alpha=0.842$ for the Somatic-Anxiety factor and $\alpha=0.666$ for the Guilt factor. The Cronbach's values for the BDI-II 3-factor solution suggest acceptable or good reliabilities.

\section{Discussion}

In our study, the 2-factor structure obtained with pregnant women included a Cognitive-Affective dimension (Factor 1) and a Somatic-Anxiety dimension (Factor 2), with an item content similar to the one obtained in other studies with nonclinical samples, except for the items irritability, agitation, crying and loss of interest in sex that were now part of the Somatic-Anxiety factor instead of the Cognitive-Affective factor. Salamero et al. [26] also obtained a similar structure with a 2-factor solution in a large sample of pregnant women, using the original version of BDI: (1) Cognitive-Affective factor and (2) Somatic and Inhibition factor.

The 2-factor solution obtained in postpartum was similar to the one found in pregnancy: (1) Somatic-Anxiety factor and (2) Cognitive-Affective factor. The items included in each factor were almost the same as in pregnancy (except the item "indecisiveness" which loaded in the Somatic-Anxiety factor instead of the Cognitive-Affective factor).

Considering the 2-factor solution it was observed that factors weight diverged within the 2 contexts. In pregnancy, more of the total variance was explained by the CognitiveAffective factor than by the Somatic-Anxiety factor $(26.5 \%$ vs. $15.5 \%$ ), while in postpartum both factors explained similar parts of the total variance $(21.3 \%$. vs. $21.4 \%)$. Similar results are usually obtained with clinical samples [4,27,28]. These results may suggest that in postpartum the Somatic-Anxiety symptoms which are often underestimated and considered "normal" may have more clinical significance and their presence should be systematically evaluated by health professionals.

When considering the 3-factor structure it was observed that in pregnancy, the Cognitive-Affective factor remained the same as in the previous 2-factor structure, but the Somatic-Anxiety factor was divided in an Anxiety factor and in a Fatigue factor. This factor solution is new and particularly interesting as it separates anxiety symptoms from common fatigue symptoms which are often observed in late pregnancy $[7,15]$. The relevance of this distinction is important in the way that a more clear-cut line between normal and pathological phenomena can be drawn, improving the study of the impact of anxiety symptoms/disorders in pregnancy which are considered a risk factor for Postpartum Depression [6,23,25]. The strong point of the present factor structure is that the cluster of symptoms associated with the Anxiety factor could eventually help predicting postpartum emotional disorders, namely Depression, in future studies.

The Cognitive-Affective factor was robust in pregnancy and remained invariable whether a 2-factor or a 3-factor model was considered. In fact, Huffman et al. [16] observed in a small sample of women $(N=62)$ who filled in the original BDI in late pregnancy and at 12 weeks postpartum that the Cognitive-Affective factor in pregnancy was the most sensitive cluster to discriminate women with low risk and at risk of Postpartum Depression.

With respect to the 3-factor model in postpartum, a small number of items belonging to the Cognitive-Affective factor in pregnancy formed a distinct factor, "Guilt" (Factor 3), composed by punishment feelings, past failure and guilt items. The Somatic-Anxiety factor was identical to the one obtained with the 2-factor model structure (included the same items) but it was the second factor obtained (after the CognitiveAffective factor) while in the 2-factor model it was the first factor found.

The 3-factor structure found in this study was not similar to the one obtained in Mahmud et al. study [19]. The authors identified a first factor, Affective, a second factor, Somatic, and a third factor, Cognitive. One explanation for the factor structure content discrepancy and factor order difference could be related to the time frame considered for subject's recruitment. In fact, in the present study we had more homogeneity in the sense that most mothers were within the 10-14 weeks after delivery, reflecting a more narrow time frame, whereas in the study by Mahmud et al. [19] Malaysian women were included in a more wide time frame (between 4 and 12 weeks after delivery) and for this reason, the dispersion may explain the factor order and content of the BDI-II (first the Affective and Somatic factors and then the Cognitive factor). Both approaches are important, in terms of the clarification of finetuning the definition of different clusters of depressive symptoms across different time-points in the postpartum period. 
In this context, the 3-factor solution for the postpartum period in our view is of significant importance as it shows a distinct and novel factor: "Guilt". Some authors [24] refer that guilt and emotional liability are depressive symptoms which could be more prominent and specific in this time. It is quite common that in the postpartum period women, particularly primipara (and most of those included in our study are primipara) experience some doubts about their role and ability of being a good mother or taking care of the baby [8,9]. Feelings of guilt such as not being a good-enough mother could be a pathological extension of those normal doubts. Postpartum Depression is not a specific mental disorder as defined in DSM-IV [1]. However, we cannot rule out the possibility that depressive symptoms such as guilt comprise a specific and important cognitive depressive content, being its identification of relevance, in terms of identification of Postpartum Depression. Therefore, its clinical and therapeutic implications should be addressed and investigated in future studies.

With respect to the study limitations, although our samples were obtained from the local community (health medical centres and general practice facilities) it is not possible to guarantee that they are truly representative of the general Portuguese population. Additionally, the BDI-II factor structures obtained in postpartum could have been affected by the fact that half of the sample answered the BDI-II during pregnancy. Beck et al. [4] referred that repeated administrations of the BDI can result on lower scores in subsequent administrations. Whether these lower scores interfered with the factor analyses obtained could not be ascertain. A crosssectional study with a large sample of Portuguese women who only answer the BDI-II in postpartum would help clarify this effect. Finally, it is not possible to determine if the results from the present study can be generalised to clinical samples. Once more the limited number of women with depression in our samples was insufficient to perform the statistical analyses required to explore this issue.

\section{Conclusion}

In summary, the 3-factor solution of the BDI-II seems to provide an interesting framework to study depressive symptomatology in pregnancy and postpartum. In pregnancy symptoms of anxiety have emerged as a single factor (Factor 2 ) independent from symptoms of fatigue (Factor 3) which are common in late pregnancy $[7,13]$ and from symptoms associated with the Cognitive-Affective dimension (Factor 1). As Psychological Distress in Pregnancy has been associated with later Postpartum Depression [25], the Anxiety factor found in this study might be helpful predicting Postpartum Depression. In postpartum besides the CognitiveAffective factor and the Somatic-Anxiety factor, a Guilt factor, identified in this research, could eventually be related to the context specificity of the depressive symptomatology in postpartum. Future studies with the 3-factor solution of the BDI-II in pregnancy and postpartum will help to clarify these issues.

\section{Acknowledgments}

We are deeply grateful to all pregnant women and mothers who participated in the present study. To doctors, nurses and administrative personnel we would equally like to thank their collaboration. This work was supported by the Portuguese Science and Technology Foundation, FCT (Project Reference: POCI 2010/FEDER/SAU-ESP/57068/2004).

\section{References}

[1] American Psychiatric Association. Diagnostic and statistical manual of mental disorders, DSM-IV. 4th ed. Washington, DC: APA; 1994.

[2] Azevedo MH, Soares MJ, Coelho I, Dourado A, Valente J, Macedo A, et al. Using consensus OPCRIT diagnoses. Br J Psychiatry 1999;175: 154-7.

[3] Azevedo MHP, Valente J, Macedo A, Dourado A, Coelho I, Pato M, et al. Versão portuguesa da "entrevista diagnóstica para estudos genéticos". Psiq Clín 1993;14:213-7.

[4] Beck AT, Steer RA, Brown GK. BDI-II manual for the Beck Depression Inventory-II. San Antonio: The Psychological Corporation; 1996.

[5] Beck AT, Ward CH, Mendelson M, Mock J, Erbaugh J. An inventory for measuring depression. Arch Gen Psychiatry 1961;142(5):559-63.

[6] Beck CT. Predictors of postpartum depression - an update. Nurs Res 2001;5:275-84.

[7] Bennett HA, Einarson A, Taddio A, Koren G, Einarson TR. Depression during pregnancy: overview of clinical factors. Clin Drug Investig 2004; 24(3):157-79.

[8] Brockington IF. Motherhood and mental health. Oxford: Oxford University Press; 1996.

[9] Buist A, Ross LE, Steiner M. Anxiety and mood disorders in pregnancy and the post-partum period. In: Castle DJ, Kulkarni J, Abel KM, editors. Mood and anxiety disorders in women. Cambridge: Cambridge University Press; 2006. p. 163-84.

[10] Coelho R, Martins A, Barros H. Clinical profiles relating gender and depressive symptoms among adolescents ascertain by the Beck Depression Inventory-II. Eur Psychiatry 2002;17:222-6.

[11] Cohen J. A power primer. Psychol Bull 1992;112:155-9.

[12] DeVellis FR. Scale development. Theory and applications. London: Sage Publications; 1991.

[13] Evans J, Heron J, Francomb H, Oke S, Golding J. Cohort study of depressed mood during pregnancy and after childbirth. BMJ 2001;323: 257-60.

[14] Green SB, Salkind NJ, Akey TM. Using SPSS for Windows: analysing and understanding data. 2nd ed. New Jersey: Prentice Hall; 1999.

[15] Halbreich U. Prevalence of mood symptoms and depression during pregnancy: implications for clinical practice and research. CNS Spectr 2004;9:177-84.

[16] Huffman LC, Lamour M, Bryan YE, Pederson FA. Depressive symptomatology during pregnancy and the postpartum period: is the Beck Depression Inventory applicable? J Reprod Infant Psychol 1990; 8:87-97.

[17] Johanson R, Chapman G, Murray D, Johnson I, Cox J. The North Staffordshire Maternity Hospital prospective study of pregnancy-associated depression. J Psychosom Obstet Gynaecol 2000;21:93-7.

[18] Kaelber CT, Moul DE, Farmer ME. Epidemiology of depression. In: Beckham EE, Leber WR, editors. Handbook of depression. NewYork: Guilford Press; 1995. p. 3-35.

[19] Mahmud W, Awang A, Herman I, Mohamed M. Analysis of the psychometric properties of the Malay version of Beck Depression Inventory-II (BDI-II) among postpartum women in Kedah, North West of Peninsular Malaysia. Malaysian J Med Sci 2004;11(2):19-25.

[20] Martins A, Coelho R, Ramos E, Barros H. Administração do BDI-II a adolescentes portugueses: resultados preliminares. Rev Port Psicossom 2000;2(1):123-32. 
[21] McGuffin P, Farmer A, Harvey I. A polydiagnostic application of operational criteria in studies of psychotic illness. Arch Gen Psychiatry 1991; 48:764-70.

[22] Nurnberger JL, Blehar MC, Kaufmann CA, York-Cooler C, Simpson SG, Harkavy-Friedman J, et al. Diagnostic interview for genetic studies: rationale, unique features and training. Arch Gen Psychiatry 1994;51:849-59.

[23] O'Hara MW, Swain AM. Rates and risk of postpartum depression-a meta analysis. Int Rev Psychiatry 1996;8:37-54.

[24] Riecher-Rössler A, Rohde A. Diagnostic classification of perinatal mood disorders. In: Riecher-Rössler A, Steiner M, editors. Perinatal stress, mood and anxiety disorders: from bench to bedside. Basel: Karger; 2005. p. 6-27.

[25] Robertson E, Celasun N, Stewart DE. Risk factor for postpartum depression. In: Stewart DE, Robertson E, Dennis CL, Wallington T, editors. Postpartum depression: literature review of risk factors and interventions. Toronto: University Health Network; 2003.

[26] Salamero M, Marcos T, Gutiérrez F, Rebull E. Factorial study of the BDI in pregnant women. Psychol Med 1994;24:1031-5.
[27] Steer RA, Ball R, Ranieri WF, Beck AT. Dimensions of the Beck Depression Inventory-II in clinical depressed outpatients. J Clin Psychol 1999;55:117-28.

[28] Steer RA, Kumar G, Ranieri WF, Beck AT. Use of the Beck Depression Inventory-II with adolescent psychiatric outpatients. J Psychopathol Behav Assess 1998;20(2):127-37.

[29] Storch EA, Roberti JW, Roth DA. Factor structure, concurrent validity and internal consistency of the Beck Depression Inventory - second edition in a sample of college students. Depress Anxiety 2004;19:187-9.

[30] Ward L. Comparison of factor structure models for the Beck Depression Inventory-II. Psychol Assess 2006;18(1):81-8.

[31] Whisman MA, Perez JE, Ramel W. Factor structure of the Beck Depression Inventory - second edition (BDI-II) in a student sample. J Clin Psychol 2000;56(4):545-51.

[32] World Health Organization - WHO. The ICD-10 classification of mental and behavioural disorders. Clinical descriptions and diagnostic guidelines. Geneva: World Health Organization; 1992. 